International Journal of

Environmental Research and

Public Health

ISSN 1660-4601

www.mdpi.com/journal/ijerph

Article

\title{
Acoustic Noise Alters Selective Attention Processes as Indicated by Direct Current (DC) Brain Potential Changes
}

\author{
Karin Trimmel ${ }^{1}$, Julia Schätzer ${ }^{2}$ and Michael Trimmel ${ }^{2,3, *}$ \\ 1 Department of Neurology, Medical University of Vienna, Vienna 1090, Austria; \\ E-Mail: karin.trimmel@meduniwien.ac.at \\ 2 Department of Psychology, University of Vienna, Vienna 1010, Austria; \\ E-Mail: julia.schaetzer@gmail.com \\ 3 Institute for Environmental Hygiene, Center for Public Health, Medical University of Vienna, \\ Vienna 1090, Austria
}

* Author to whom correspondence should be addressed; E-Mail: michael.trimmel@ meduniwien.ac.at; Tel.: +43-1-40160-34911; Fax: +43-1-40160-934903.

External Editor: Paul B. Tchounwou

Received: 23 June 2014; in revised form: 2 September 2014 / Accepted: 16 September 2014 / Published: 26 September 2014

\begin{abstract}
Acoustic environmental noise, even of low to moderate intensity, is known to adversely affect information processing in animals and humans via attention mechanisms. In particular, facilitation and inhibition of information processing are basic functions of selective attention. Such mechanisms can be investigated by analyzing brain potentials under conditions of externally directed attention (intake of environmental information) versus internally directed attention (rejection of environmental stimuli and focusing on memory/planning processes). This study investigated brain direct current (DC) potential shifts - which are discussed to represent different states of cortical activation — of tasks that require intake and rejection of environmental information under noise. It was hypothesized that without background noise rejection tasks would show more positive DC potential changes compared to intake tasks and that under noise both kinds of tasks would show positive DC shifts as an expression of cortical inhibition caused by noise. DC potential shifts during intake and rejection tasks were analyzed at 16 standard locations in 45 persons during irrelevant speech or white noise vs. control condition. Without noise, rejection tasks were associated with more positive DC potential changes compared to intake tasks. During
\end{abstract}


background noise, however, this difference disappeared and both kinds of tasks led to positive DC shifts. Results suggest-besides some limitations - that noise modulates selective attention mechanisms by switching to an environmental information processing and noise rejection mode, which could represent a suggested "attention shift". Implications for fMRI studies as well as for public health in learning and performance environments including susceptible persons are discussed.

Keywords: acoustic noise; facilitation and inhibition; direction of attention; brain DC potentials; attention control; attention shift

\section{Introduction}

Environmental acoustic noise (even of low to moderate intensity) is known to have- - besides health effects [1-7] — a number of adverse effects on human information processing by modulating attention [8-11]. The function of attention has already been described by James [12] "Every one [sic] knows what attention is. It is the taking possession by the mind, in clear and vivid form, of one out of what seem several simultaneously possible objects or trains of thought. Focalization, concentration, [sic] of consciousness are of its essence. It implies withdrawal of some things in order to deal effectively with others, and is a condition which has a real opposite in the confused, dazed, scatter-brained state, which in French is called distraction, and Zerstreutheit in German" pp. 403-404). In attention processes, facilitation of relevant information and inhibition of non-relevant stimuli are seen as basic underlying mechanisms of selective attention [13]. This means that during complex cognitive tasks, non-task-relevant information is filtered out as an expression of selective attention. Rejection of environmental stimuli would therefore take place in tasks that require attention to be directed towards internal processing or in cases where environmental stimuli would affect current information processing. Acoustic noise has often been investigated as it is part of everyday life and is therefore of high practical relevance. The present study aims to investigate the effect of acoustic background noise on an aspect of a neurophysiological indication of brain processes, namely cortical direct current (DC) potentials, which have been discussed to reflect different states of cortical activation [14-16].

In the present study, effects of facilitation and inhibition processes of selective attention were investigated by manipulating the direction of attention towards environmental stimuli or towards internal mental activity. According to Lacey [17], attention tasks that require observation of environmental stimuli are referred to as "environmental intake" tasks and attention tasks that require attention to be directed towards internal processing are labeled "environmental rejection" tasks. Neurophysiological correlates of the direction of attention have been investigated with EEG recordings [18], reporting more alpha activity during internally directed attention tasks compared to externally directed attention tasks. Additionally, higher alpha amplitudes have been observed during internally directed attention tasks $[19,20]$ which was interpreted as an expression of inhibition of non-task-relevant information. However, currently no data exist to examine whether direction of attention modulates brain DC potential shifts, and whether this interacts with acoustic environmental background noise, which poses the topic of the present study. 
The origins of DC potentials and their shifts are-besides some contribution by glial cells [21,22]considered to be mainly neuronal representing hypo- and hyperpolarization of pyramidal cells at their apical dendrites with underlying complex interactions between the cortex, thalamus, reticular formation, and the basal ganglia [21-23]. DC potential shifts may also be altered by changes in partial pressure of carbon dioxide $\left(\mathrm{pCO}_{2}\right)$ [24] and therefore by respiration rates [25,26]. However, recent fMRI findings [27] support the view that changes in DC potentials by investigating infra-slow fluctuations (ISFs) in scalp potentials reflect changes in cortical excitability and still have functional significance on the execution of cognitive tasks [28,29].

It has been discussed that negative DC potential shifts recorded from the scalp [15,30,31] as well as from single cell recordings [32] are a sign of higher excitability and therefore reflect cortical activation, which is associated for instance with better performance and shorter reaction times [14,30]. In addition, there is also some evidence that facilitation of processing of environmental information is associated with preceding negative DC potential shifts [31] and moreover, higher sustained negative DC potentials were found in association with shorter reaction times [33].

In contrast, positive DC potential shifts, which might occur during sleep [34], but also during processes of selective attention, are seen as a sign of reduced excitability or cortical inhibition $[15,35,36]$. In regard to noise, Trimmel and Poelzl [9] found more frontal positivity and less parietal negativity for a spatial-cueing paradigm [37] under acoustical noise conditions (i.e., a mixture of environmental low intensity sounds) compared to no-noise conditions, which was interpreted as an expression of filtering out irrelevant information.

Therefore, in the present study it was hypothesized that brain DC potentials differ for intake and rejection tasks and that rejection tasks are accompanied by more positive DC potential shifts compared to intake tasks. It was furthermore expected that under noise conditions, intake tasks would also be associated with more positive DC potentials compared to no-noise conditions as an expression of rejection of background noise. In order to take into account modality effects [38,39], intake and rejection tasks were both investigated by verbal and visual figural tasks. Noise effects were investigated by both irrelevant speech and white noise to give some indication of the generality of noise effects.

\section{Material and Methods}

\subsection{Participants}

Forty-eight participants (30 females) with an age range of 19-37 years (mean age and SD $24 \pm 5$ years) participated in the study. Participation was voluntary (for course credit) and all persons were right-handed, non-medicated and had normal or corrected-to-normal vision. The experiment was ethically and formally approved by the University of Vienna and informed consent was obtained from the participants.

\subsection{Design}

The study was based on a 2 (Noise Type; irrelevant speech $v s$. white noise) $\times 2$ (Noise Condition; noise $v s$. no-noise) $\times 2$ (Direction of Attention; intake $v s$. rejection) $\times 2$ (Modality of attended stimuli; figural $v s$. verbal) $\times 16$ (Recording Location) ANOVA design with the last four factors as repeated 
measures. The sequences of the four attention tasks (intake-figural, intake-verbal, rejection-figural, rejection-verbal) as well as of noise condition were balanced across participants. The sequence of the attention tasks was kept constant within persons for the noise and the no-noise condition.

\subsection{Recordings}

DC potentials were recorded with a BioSemi system (Amsterdam, Netherlands) using pin-type sintered $\mathrm{Ag} / \mathrm{AgCl}$ electrodes. Electrode gel was applied to the holders in the headcap and the electrodes were clicked into their holders. In order to avoid artifacts resulting from instabilities caused by the electrolyte-skin-interface [40], recordings were performed at least 60 minutes after electrode attachment. EEG was recorded from frontopolar (Fp1, Fp2), frontal (F3, Fz, F4), central (C3, Cz, C4), parietal (P3, $\mathrm{Pz}, \mathrm{P} 4)$, occipital (O1, Oz, O2), and temporal (T7, T8) locations (according to the 10-20-system), referenced to the CMS-DRL ground and re-referenced off-line to linked mastoids. Recordings were collected from DC to $30 \mathrm{~Hz}$ and digitized with a sample rate of $512 \mathrm{~Hz}$. In addition, vertical EOG (above and below the left eye) as well as skin potential (from the palmar side of the left index finger to the extensor side of the forearm) were obtained as control recordings with the same filter settings as for EEG recordings.

\subsection{Experimental Conditions}

\subsubsection{Noise}

All 48 participants experienced a noise condition and a control condition counterbalanced. In the noise condition, 24 persons experienced white noise and 24 persons experienced irrelevant speech (see Table 1 for details of sound pressure levels [SPLs]). Irrelevant speech was presented by playing backwards a CD of an Austrian comedian (Josef Hader, "Privat", Audio CD 1995).

Table 1. Measures of the SPLs for noise conditions and control condition.

\begin{tabular}{ccc}
\hline & Leq dB (A) & max dB (A) \\
\hline Irrelevant Speech & & \\
\hline Fast & 61.5 & 71.6 \\
Slow & 61.6 & 66.8 \\
\hline White Noise & & \\
\hline Fast & 68.7 & 69.5 \\
Slow & 68.8 & 69.0 \\
\hline Control & & \\
\hline Fast & 38.2 & 48.1 \\
Slow & 37.6 & 41.8 \\
\hline
\end{tabular}

\subsubsection{Attention Tasks}

Two externally directed (i.e., intake) and two internally directed (i.e., rejection) attention tasks [17] with one figural and one verbal task each were developed to investigate the effects of the direction of attention with the consideration of modality. Each task consisted of two parts: the computerized attention 
task and the examination of task execution, during which the participants were filling in answer sheets in order to verify that the instructions for the attention tasks had been followed.

Intake Verbal- “Listening Task”

A memory exercise about a fictitious state in Africa, taken from the Lern- und Merkfähigkeitstest [41], a German memory test, was played over a period of 90 seconds, and participants were asked to listen attentively and try to memorize as many facts as possible.

Intake Figural—“Recognizing Photographs of Trees"

Participants were presented 10 photographs of various trees, displayed over approximately $75 \%$ of the computer screen, over a period of $90 \mathrm{~s}$, with a presentation time of $9 \mathrm{~s}$ for each photograph. Participants were instructed to try to remember those photographs.

Reject Verbal—“Creating Words"

A syllable taken from the Verbaler Kreativitäts-Test [42], a German verbal creativity test, was presented to participants for $90 \mathrm{~s}$ and they were asked to create as many words as possible beginning with the respective presented syllable and to try to remember those words.

Reject Figural_-"Picture Completion”

Participants were presented four incomplete figures taken from the "Picture Completion" task from the Torrance Tests of Creative Thinking [43]. Persons were instructed to complete and memorize those figures for $90 \mathrm{~s}$.

\subsection{Procedure}

Participants were seated in front of a laptop $\left(\right.$ ACER $^{\odot}$ Travelmate $291 \mathrm{LCi}$ ) with a distance of $80 \mathrm{~cm}$ between the person and the laptop screen. The visual angle of the screen measured $20^{\circ}$ horizontally and $15^{\circ}$ vertically. Prior to the presentation of the attention tasks, persons were informed about the experimental procedure, all attention tasks were explained, and test trials were performed. The experimental procedure started with participants sitting in front of a grey computer screen for one minute with the last $30 \mathrm{~s}$ serving as baseline for the first attention task. Each attention task then began with an instructional announcement of the respective task on the computer screen for $20 \mathrm{~s}$, followed by the attention task itself, which lasted $90 \mathrm{~s}$. After the task, examination of task execution took place, lasting $90 \mathrm{~s}$ as well. Subsequently, participants sat for $30 \mathrm{~s}$, this epoch served as baseline for the following attention task. In the noise condition, white noise or irrelevant speech was presented continuously for the whole block of the four attention tasks.

\subsection{Analysis}

EEG data were exported with the program BESA (MEGIS Software GmbH, Gräfelfing, Germany) and mean voltage values for each one second window were calculated. DC potential changes were 
exported for epochs of $230 \mathrm{~s}$, namely $30 \mathrm{~s}$ baseline, $20 \mathrm{~s}$ of instruction, $90 \mathrm{~s}$ of the attention task, and $90 \mathrm{~s}$ of the examination of task execution. DC drift artifact correction was performed according to Hennighausen, Heil, and Rösler [44] over a period of $260 \mathrm{~s}$ (the epoch of $230 \mathrm{~s}$ described above plus $30 \mathrm{~s}$ of baseline of the following task) and the first and last $60 \mathrm{~s}$ of each 260 -s-epoch were used to calculate the slope and eliminate it from the recorded segment in each recording channel. The mean DC potentials from baseline were set to $0 \mu \mathrm{V}$ and mean values of the attention tasks were calculated for the statistical analysis of the DC potential shifts during the attention tasks.

Statistical analysis was performed with Statistica (Version 7, StatSoft Inc., Tulsa, OK, USA) and an alpha level of $p=0.05$ was applied for all statistical tests. MANOVA was performed for DC potentials with the factors location (Fp1, Fp2, F3, Fz, F4, C3, Cz, C4, P3, Pz, P4, T7, T8, O1, Oz, O2), direction of attention (intake vs. rejection), modality (figural $v s$. verbal), and noise condition (no-noise $v s$. noise) as within factors and with noise type (irrelevant speech $v s$. white noise) as the between groups factor. For EOG and skin potential, the same analyses as for DC potentials were performed, however, without the factor location. Three persons had to be excluded from analyses because of recording artifacts.

\section{Results}

Grand means of DC potential changes as well as of vertical EOG and of skin potential are displayed in Figures 1-3. MANOVA results showed a main effect of Location $(F(15,29)=8.41, p<0.001)$ and Direction of Attention $(F(1,43)=4.51, p=0.039)$. MANOVA also showed an interaction between Noise and Location $(F(15,29)=2.07, p=0.044)$, indicating more positive DC potential changes in the noise condition compared to the control condition (as can be seen in Figures 1-3). In detail, significant effects (as indicated by LSD test) were observed at electrodes $\mathrm{Cz}, \mathrm{T} 8, \mathrm{P} 3, \mathrm{Pz}, \mathrm{P} 4, \mathrm{O} 1, \mathrm{Oz}$, and O2. Furthermore, the interaction of Noise $\times$ Direction of Attention $(F(1,43)=4.46, p=0.040)$ indicates that in the control condition rejection tasks are associated with more positive DC potential changes compared to intake tasks (Figures 1 and 4), in the noise condition, however, DC shifts of intake tasks became more positive and reached the level of rejection tasks (Figures 1-4). A trend for an effect of modality was observed in the interaction of Location $\times$ Modality $(F(15,29)=1.82, p=0.080)$, with confidence intervals indicating more positive DC potentials for verbal tasks compared to figural tasks at electrode P3. No other effects or interactions were statistically significant. Analysis of EOG revealed one statistically significant effect for Noise $(F(1,43)=7.73, p=0.008)$, indicating lower values in the noise condition compared to the control condition (Mean \pm SD: $30.09 \pm 13.90$ in the noise condition vs. $77.80 \pm 14.08$ in the control condition). No such effect was observed in frontopolar locations $(F(1,43)<1, p=$ n.s. $)$ which suggests that confounding of EEG recordings by EOG is unlikely. For skin potential, the only significant effect was a main effect of Noise Type $(F(1,43)=13.79, p<0.001)$ with higher values in the irrelevant speech group compared to the white noise group (Mean \pm SD: $601.33 \pm 197.04$ for irrelevant speech $v s .-422.18$ \pm 192.71 for white noise). No such effect was observed for pooled EEG recordings $((F(1,43)=1.24, p$ $=0.27)$; Mean \pm SD: $35.20 \pm 11.42$ for irrelevant speech $v s .17 .41 \pm 11.17$ for white noise). 
Figure 1. No-noise condition: Grand means of brain DC potentials (positivity upward) as well as vertical EOG and skin potential for the four attention task conditions. The timeline indicates when instruction (seconds 1-20), attention tasks (seconds 21-110; between the vertical broken lines), and examination of task execution (seconds 111-200) took place.
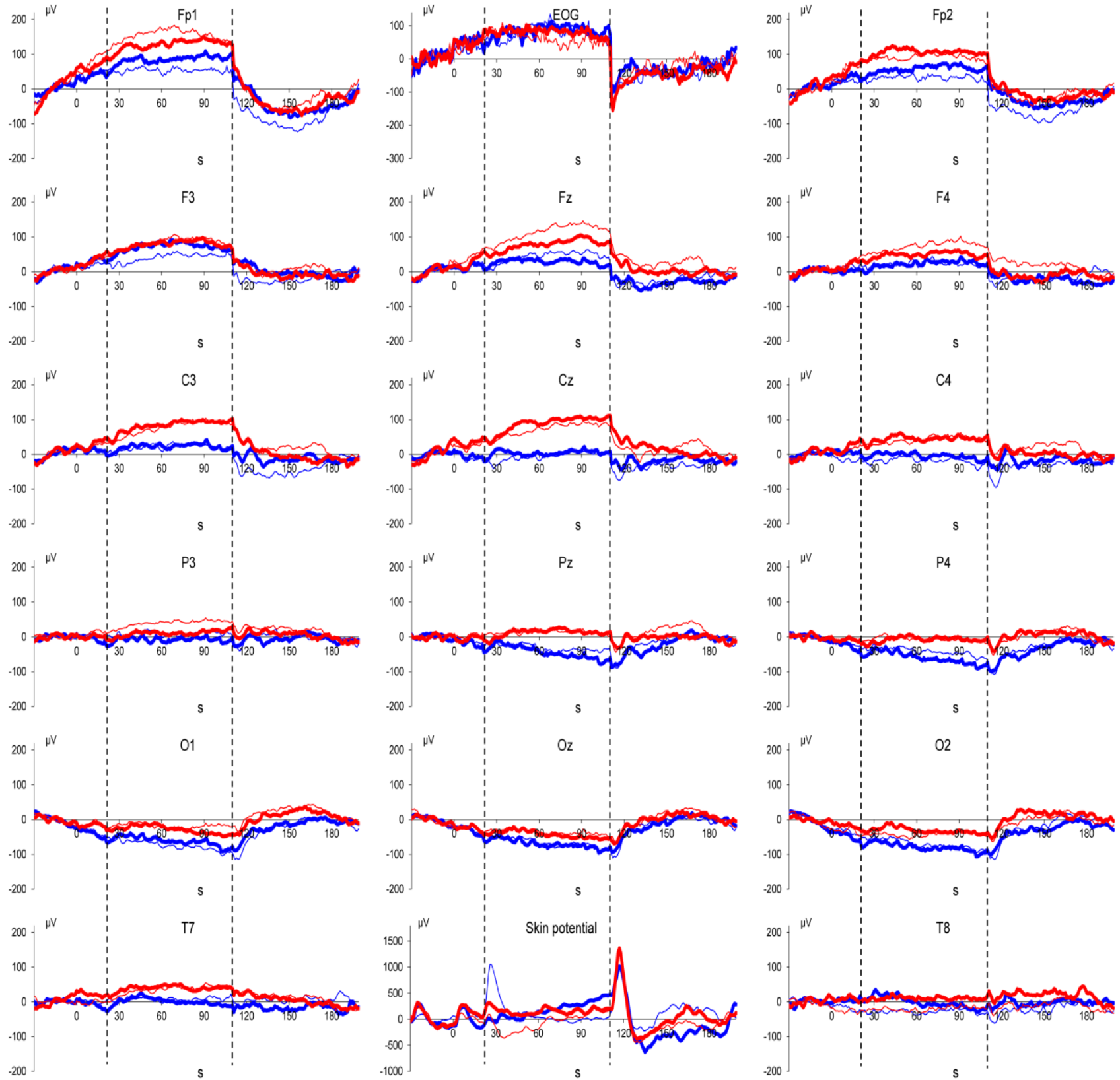
Figure 2. Irrelevant speech condition: Grand means of brain DC potentials (positivity upward) as well as vertical EOG and skin potential for the four attention task conditions. The timeline indicates when instruction (seconds 1-20), attention tasks (seconds 21-110; between the vertical broken lines), and examination of task execution (seconds 111-200) took place.

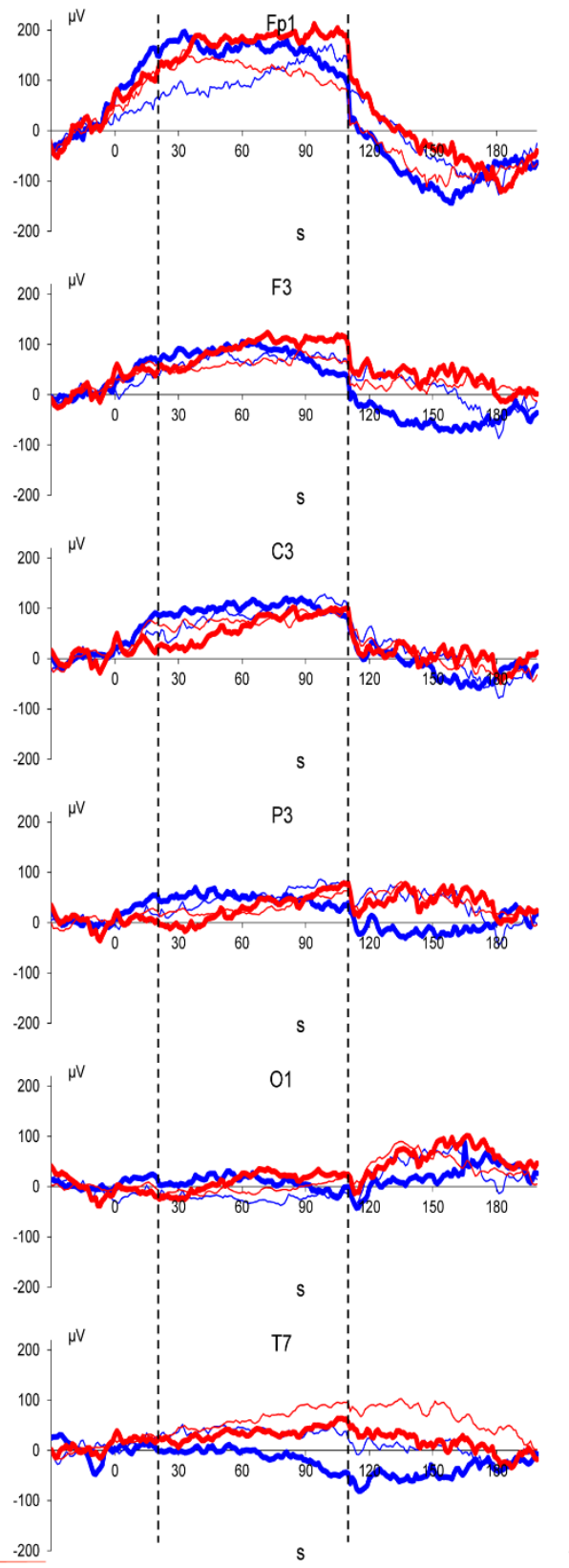

—ntake figural

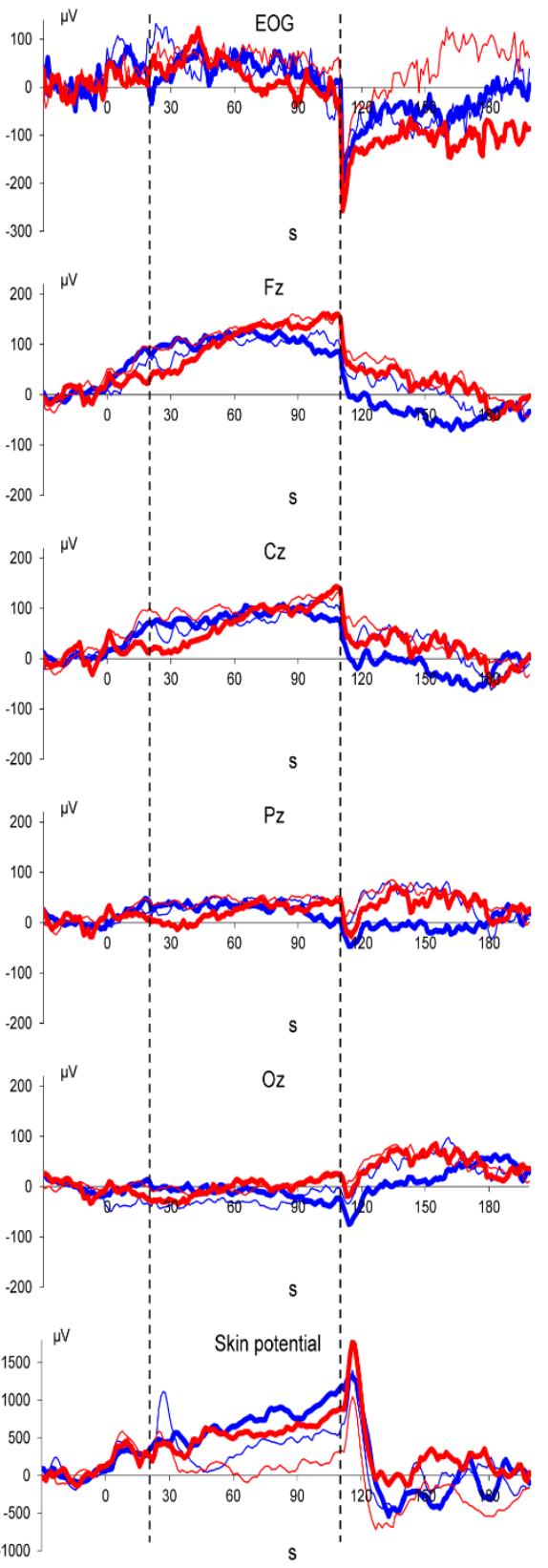

- Intake verbal Rejection figural

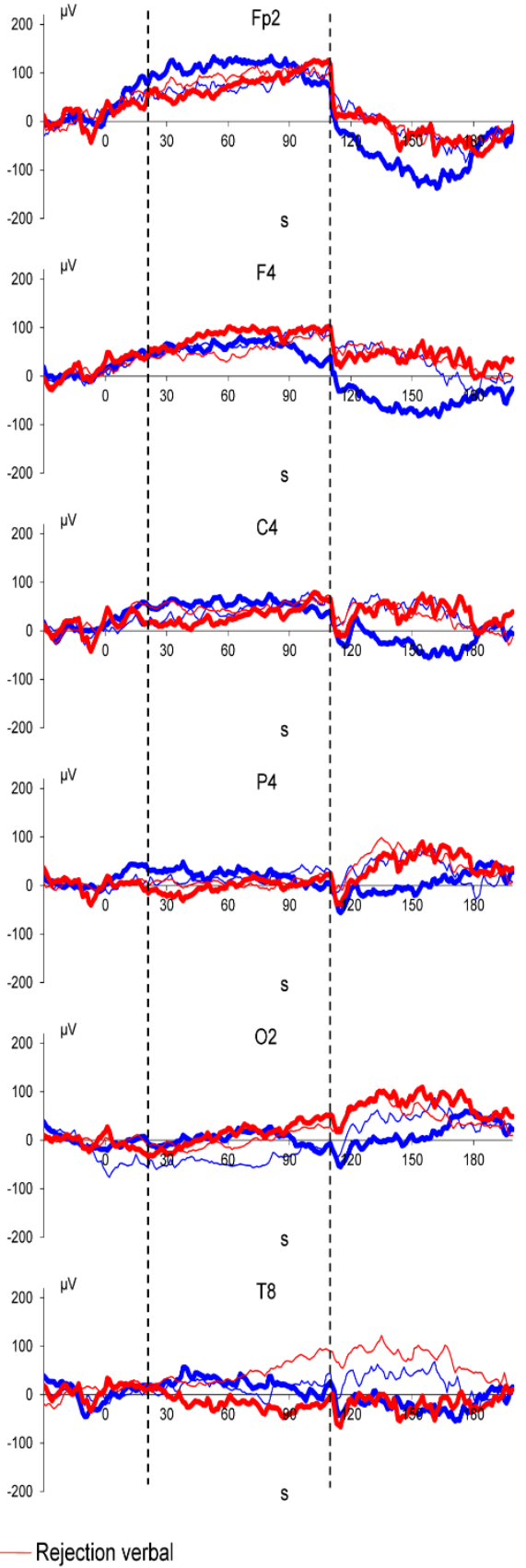


Figure 3. White noise-condition: Grand means of brain DC potentials (positivity upward) as well as vertical EOG and skin potential for the four attention task conditions. The timeline indicates when instructions (seconds 1-20), attention tasks (seconds 21-110; between the vertical broken lines), and examination of task execution (seconds 111-200) took place.
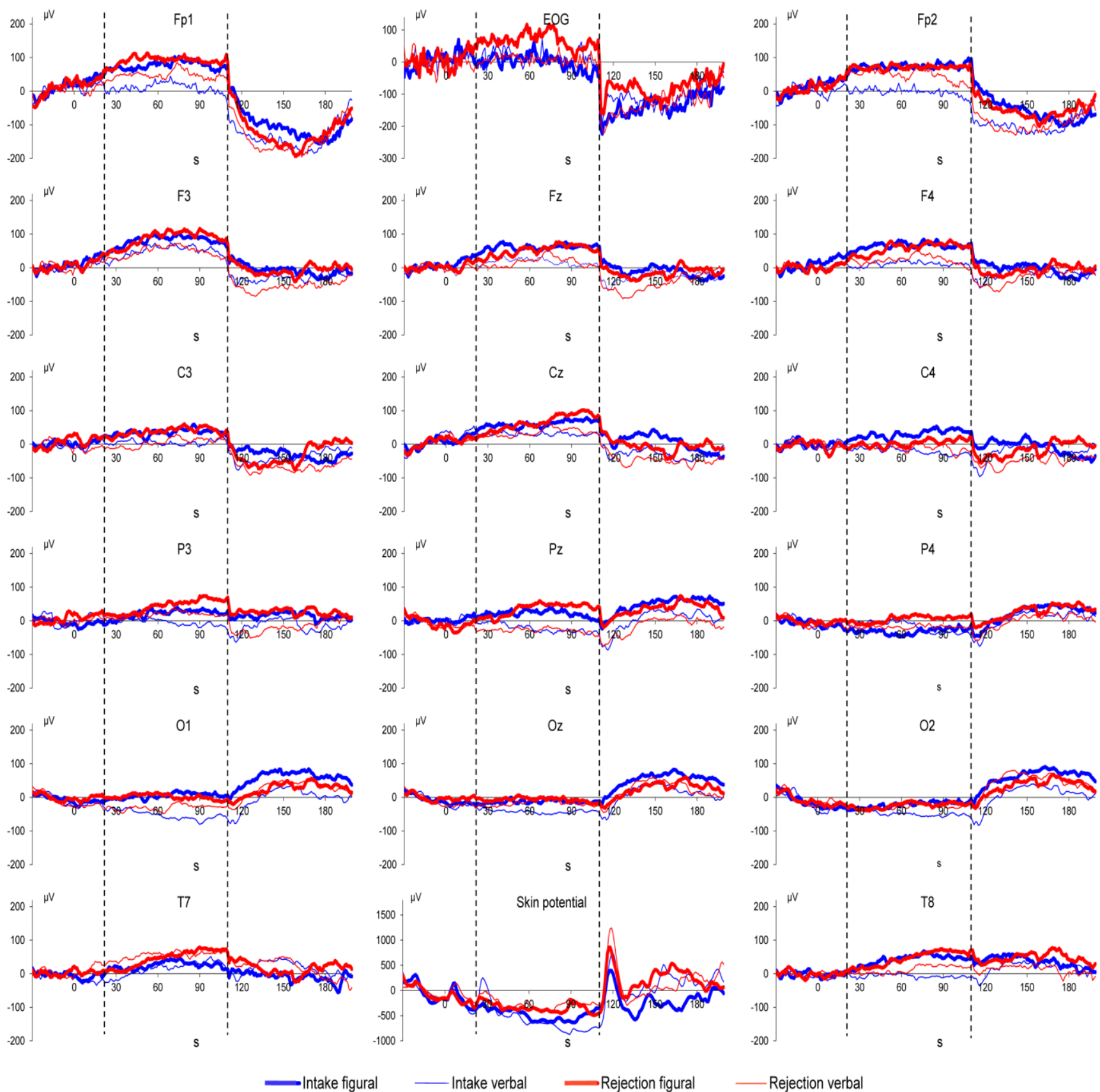
Figure 4. Mean values $( \pm 95 \% \mathrm{CI})$ of pooled brain DC potentials for intake and rejection tasks in control condition and noise condition.

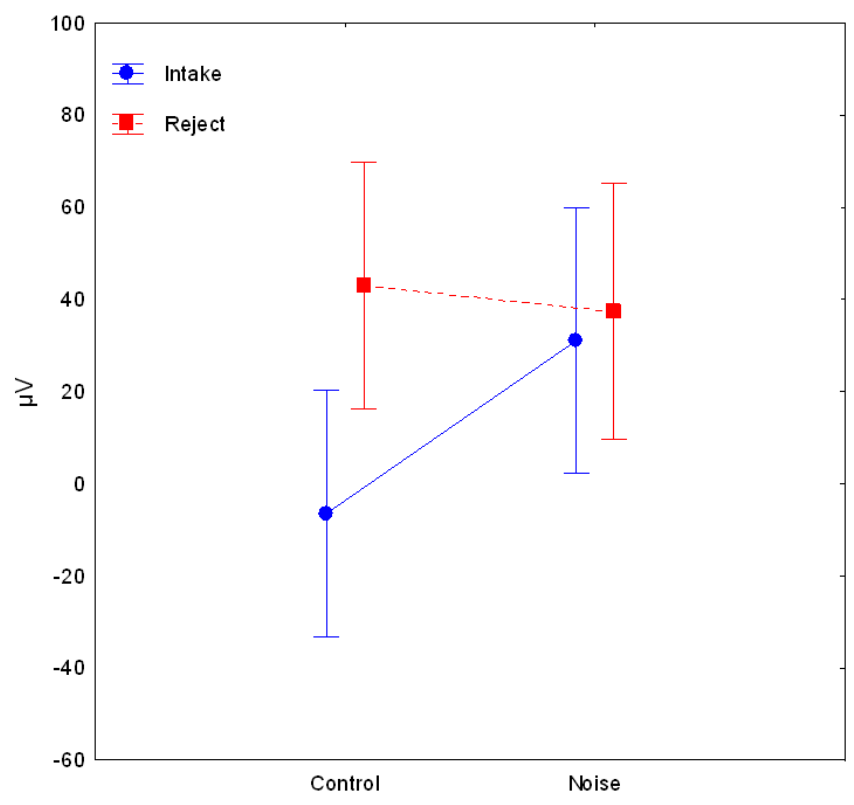

\section{Discussion}

Results indicate different brain DC potentials for intake and rejection tasks in the control condition. It could be shown that without noise, tasks requiring internally directed attention showed more positive DC shifts compared to externally directed attention tasks. Under noise conditions, however, this difference disappeared since environmental rejection tasks as well as intake tasks were associated with positive DC potential shifts. Analyses of control recordings (EOG, skin potential) revealed different patterns compared to effects of brain DC potentials, which makes confounding of the obtained results of brain DC potentials unlikely. However, possible confounding of the results due to the lack of control for blood brain barrier $\mathrm{pCO}_{2}$ changes cannot be completely ruled out.

It is assumed that, as an expression of selective attention, information that is not relevant to a task will be inhibited in order to facilitate the processing of relevant information [13]. In the present study, DC shifts towards positivity are suggested to reflect this inhibition (i.e., rejection) process. This view is supported by studies investigating DC potential shifts both from scalp recordings [15,31] as well as from single cell recordings [32,35], which suggest surface positive DC potential changes to reflect cortical inhibition.

The effect that direction of attention as intake $v s$. rejection is associated with different/altered electrocortical activity was also found analyzing alpha activity $[19,20]$. Results of this study suggest that when trying to filter non-relevant environmental information, participants performing intake tasks show positive DC potential shifts comparable to those of participants performing rejection tasks. This could be interpreted as an additional cognitive load caused by acoustic background noise, which leads to altered information processing of intake tasks as an expression of an inhibition of environmental noise. This interpretation is supported by an investigation on the effects of low intensity background noise on DC potentials of an attention task, where positive shifts were interpreted as a "perceptual defense" response to noise [9]. Furthermore, positive shifts recorded directly from the brain in rat experiments were discussed to represent "protective inhibition" [45] of a "death cry" of another rat being snapped by 
a snake. The view that top-down processes as expressed by the direction of attention alter cortical activity by means of facilitation/inhibition according to the task on hand is supported by Ghatan et al. [46] investigating effects of irrelevant speech by fMRI, where noise-dependent changes in cerebral blood flow were interpreted as a sign of "inhibitory modulation" of non-task-relevant information. A recent animal study (three-spined stickleback, Gasterosteus aculeatus) [47] suggests that exposure to acoustic noise results in "decreased foraging efficiency" by interfering with attention processes, our study could be interpreted as a possible display of the underlying mechanism of such an "attention shift".

Thus one can speculate that the attention shift caused by environmental noise is the underlying process for the observed harmful effects on learning, memory, and performance. There is good empirical evidence that environmental noise diminishes cognitive learning and memory performance in school classes [48-53]. Such an effect would be predicted by this study, because the outcome of our investigation displays a change of the brain DC potential towards positivity in intake tasks during noise. It is well established that positive brain DC potentials, as an expression of cortical inhibition, are associated with lowered performance in cognition and motor behavior [54,55]. Moreover, in intake tasks during noise an additional mental process seems to be active, namely an environmental noise rejection process, representing an additional mental task load. This then may be associated with extra mental effort (as supported by the observation of the coincidence of positive DC shifts with task load [56,57]) caused by task switching as recently suggested in a theoretical approach [58]. The higher mental effort for learning during environmental background noise was recently supported by the analysis of spontaneous skin conductance fluctuations as an indication of the activity of the sympathetic nervous system [10]. Thus there is converging evidence that environmental noise affects cognitive performance and memory processes by directing attention on environmental events. That means that an additional mental process is necessary, namely filtering out non-relevant environmental information, which in turn leads to a kind of a "dual task paradigm" if one is already engaged in a cognitive task, associated with less performance and higher mental effort. From dual task experiments it is well established that due to limitations in resources [59], the performance of cognitive modules which are engaged in two tasks of the same modality at the same time-e.g., perception of auditive information and filtering out auditive noise - shows reduced performance and needs higher mental effort compared to a single task.

A trend for an effect of modality could be observed by more positive DC potential shifts for verbal tasks compared to figural tasks at location P3. This could be interpreted as an additional challenge for the left hemisphere (i.e., the language-dominant hemisphere in right-handed persons) during verbal tasks. This trend, however, was independent of the presence of background noise. A limitation of the study can be seen by not having recorded respiration rate to rule out that the interaction of direction of attention with noise could be affected by changes in $\mathrm{pCO}_{2}$ [24-26]. However, according to Vuopio et al. [25], $\mathrm{pCO}_{2}$-induced DC changes occur in the range of minutes, whereas in our study, DC shifts occur within seconds. Furthermore, this would also imply that the respiration change only appeared for the intake tasks under noise. This argument also applies to the view that the observed effects may be caused by stress during noise. Additionally, one could argue that the figural rejection task requires both internally and externally directed attention. However, the observed noise effect appeared for figural as well as verbal tasks. Moreover, in the no-noise condition, intake and rejection tasks could be differentiated by means of DC potentials in both the figural and the verbal task, suggesting some evidence that the tasks differed in the orientation of attention. 
Practical considerations can be drawn for brain research and for public health. The present study demonstrated that noise effects modulate brain activity by mechanisms of attention, and might therefore give some theoretical background on a fundamental principle responsible for widely observed noise effects. This includes consequences for brain research methods like fMRI; as that can be associated with a considerable noisy environment, one should take into account that under such environmental noise condition altered attention mechanisms may be evoked and would bias the results as also suggested by Hommel et al. [60].

Practical consequences for public health are also obvious. (1) The attenuation and/or avoidance of environmental noise not only for health and well-being but also to avoid detrimental effects on cognitive performance and to avoid additional mental effort during cognitive tasks, in particular (2) in learning, educational, and performance environments like schools and universities [61]; (3) Furthermore, especially susceptible persons who suffer from ADHD [62], dyslexia [63], or hearing impairment [64] as well as elderly persons $[65,66]$ are in particular challenged by noise.

\section{Conclusions}

Without noise, intake and rejections tasks can be differentiated by brain DC potentials. However, this effect disappears under task conditions of environmental noise. This may indicate an attention shift in that way, that noise challenges selective attention processes by switching to an environmental information processing and noise rejection mode. Practical considerations include that fMRI studies could be biased by noise. Furthermore, regarding public health, noise in learning and performance environments (schools, universities, etc.) should be avoided/attenuated, in particular considering susceptible persons who suffer from ADHD, dyslexia, or hearing impairment and elderly persons.

\section{Acknowledgements}

The authors want to thank the reviewers and the editor for the helpful comments and for encouraging us to expand the scope of this manuscript. The authors also wish to thank all participants who took part in the study, Monika Meixner-Pendleton for her assistance with data analysis, and Gabriela Langer for her help in preparing the manuscript.

\section{Author Contributions}

Michael Trimmel was the research coordinator and supervisor. Julia Schätzer contributed to background research, research design, operationalization, data acquisition, data reduction, statistical analysis, and writing the paper. Karin Trimmel contributed to background research, research design, statistical analysis, interpretation, and writing the paper.

\section{Conflicts of Interest}

The authors declare no conflicts of interest. 


\section{References}

1. Basner, M.; Babisch, W.; Davis, A.; Brink, M.; Clark, C.; Janssen, S.; Stansfeld, S. Auditory and non-auditory effects of noise on health. Lancet 2014, 383, 1325-1332.

2. Babisch, W. The noise/stress concept, risk assessment and research needs. Noise Health 2002, 4, $1-11$.

3. Jahncke, A.; Hygge, S.; Halin, N.; Green, A.M.; Dimberg, K. Open-plan office noise: Cognitive performance and restoration. J. Environ. Psychol. 2011, 31, 373-382.

4. Maschke, C.; Niemann, H. Health effects of annoyance induced by neighbour noise. Noise Control Eng. J. 2007, 55, 348-356.

5. Lercher, P.; Botteldooren, D.; Widmann, U.; Uhrner, U.; Kammeringer, E. Cardiovascular effects of environmental noise: Research in Austria. Noise Health 2011, 13, 234-250.

6. Shepherd, D.; Welch, D.; Dirks, K.N.; McBride, D. Do quiet areas afford greater health-related quality of life than noisy areas? Int. J. Environ. Res. Public Health 2013, 10, 1284-1303.

7. Stansfeld, S.A.; Matheson, M.P. Noise pollution: Non-auditory effects on health. Br. Med. Bull. 2003, 68, 243-257.

8. Woolner, P.; Hall, E. Noise in schools: A holistic approach to the issue. Int. J. Environ. Res. Public Health 2010, 7, 3255-3269.

9. Trimmel, M.; Poelzl, G. Impact of background noise on reaction time and brain DC potential changes of VDT-based spatial attention. Ergonomics 2006, 49, 202-208.

10. Trimmel, M.; Atzlsdorfer, J.; Tupy, N.; Trimmel, K. Effects of low intensity noise from aircraft or from neighbourhood on cognitive learning and electrophysiological stress responses. Int. J. Hyg. Environ. Health 2012, 215, 547-554.

11. Hygge, S.; Boman, E.; Enmarker, I. The effects of road traffic noise and meaningful irrelevant speech on different memory systems. Scand. J. Psychol. 2003, 44, 13-21.

12. James, W. The Principles of Psychology; Holt: New York, NY, USA, 1890.

13. Milliken, B.; Tipper, S. Attention and inhibition. In Attention; Pashler, H., Ed.; Psychology Press: Hove, UK, 1998.

14. Birbaumer, N.; Elbert, T.; Canavan, A.G.; Rockstroh, B. Slow potentials of the cerebral cortex and behavior. Physiol. Rev. 1990, 70, 1-41.

15. Rösler, F.; Heil, M.; Röder, B. Slow negative brain potentials as reflections of specific modular resources of cognition. Biol. Psychol. 1997, 45, 109-141.

16. Haider, M.; Groll-Knapp, E.; Ganglberger, J.A. Event-related slow (DC) potentials in the human brain. Rev. Physiol. Biochem. Pharmacol. 1981, 88, 125-197.

17. Lacey, J.I. Psychophysiological approaches to the evaluation of psychotherapeutic process and outcome. In Research in Psychotherapy; Rubinstein, E.A., Parloff, M.B., Eds.; American Psychological Association: Washington, DC, USA, 1959.

18. Ray, W.; Cole, H. EEG alpha activity reflects attentional demands, and beta activity reflects emotional and cognitive processes. Science 1985, 228, 750-752.

19. Cooper, N.; Croft, R.; Dominey, S.; Burgess, A.; Gruzelier, J. Paradox lost? Exploring the role of alpha oscillations during externally $v s$. internally directed attention and the implications for idling and inhibition hypotheses. Int. J. Psychophysiol. 2003, 47, 65-74. 
20. Cooper, N.; Burgess, A.; Croft, R.; Gruzelier, J. Investigating evoked and induced electroencephalogram activity in task-related alpha power increases during an internally directed attention task. Neuroreport 2006, 17, 205-208.

21. Somjen, G.G. Contribution of neuroglia to extracellular sustained potential shifts. In Multidisciplinary Perspectives in Event-Related Brain Potential Research; Otto, D.A., Ed.; US Environmental Protection Agency: Washington, DC, USA, 1978; pp. 19-24.

22. Caspers, H.; Speckmann, E.J.; Lehmenkueler, A. Electrogenesis of slow potentials. In Self-Regulation of Brain and Behavior; Elbert, T., Rockstroh, B., Lutzenberger, W., Birbaumer, N., Eds.; Springer: Berlin, Germany, 1984; pp. 26-41.

23. Bauer, H.; Korunka, C.; Leodolter, M. Possible glial contribution in the electrogenesis of SPs. In Slow Potential Changes in the Human Brain; McCallum, W.C., Curry, S.H., Eds.; Plenum: New York, NY, USA, 1993; pp. 23-24.

24. Caspers, H.; Speckmann, E.J.; Lehmenkuehler, A. Effects of $\mathrm{CO}_{2}$ on cortical field potentials in relation to neuronal activity. In Origin of Cerebral Field Potentials; Speckmann, E.J., Caspers, H., Eds.; Georg Thieme: Stuttgart, Germany, 1979; pp. 151-163.

25. Vuopio, J.; Tallgren, P.; Heinonen, E.; Vanhatalo, S.; Kaila, K. Millivolt-scale DC shifts in the human scalp EEG: Evidence for a nonneuronal generator. J. Neurophysiol. 2003, 89, 2208-2214.

26. Nita, D.A.; Vanhatalo, S.; Lafortune, F.D.; Voipio, J.; Kaila, K.; Amzica, F. Nonneuronal origin of $\mathrm{CO}_{2}$-related DC EEG shifts: An in vivo study in the cat. J. Neurophysiol. 2004, 92, 1011-1022.

27. Hiltunen, T.; Kantola, J.; Abou Elseoud, A.; Lepola, P.; Suominen, K.; Starck, T.; Nikkinen, J.; Remes, J.; Tervonen, O.; Palva, S.; et al. Infra-slow EEG fluctuations are correlated with resting-state network dynamics in fMRI. J. Neurosci. 2014, 34, 356-362.

28. Monto, S.; Palva, S.; Voipio, J.; Palva, J.M. Very slow EEG fluctuations predict the dynamics of stimulus detection and oscillation amplitudes in humans. J. Neurosci. 2008, 28, 8268-8272.

29. Trimmel, M.; Mikowitsch, A.; Groll-Knapp, E.; Haider, M. Occurrence of infraslow potential oscillations in relation to task, ability to concentrate and intelligence. Int. J. Psychophysiol. 1990, 9, 167-170.

30. Stamm, J.; Rosen, S. Cortical steady potential shifts and anodal polarization during delayed response performance. Acta Neurobiol. Exp. 1972, 32, 193-209.

31. Bauer, H.; Nirnberger, G. Concept identification as a function of preceding negative or positive spontaneous shifts in slow brain potentials. Psychophysiology 1981, 18, 466-469.

32. Pirch, J.H.; Peterson, S.L. Event-related slow potentials and activity of singly neurons in rat frontal cortex. Int. J. Neurosci. 1981, 15, 141-146.

33. Trimmel, M.; Meixner-Pendleton, M. Relationship of brain DC potentials to vigilance-based ERPs and operator functional state. In Operator Functional State. The Assessment and Prediction of Human Performance Degradation in Complex Tasks; Hockey, G.R., Gaillard, A.W., Burow, O., Eds.; IOS Press: Amsterdam, The Netherland, 2003; pp. 131-139.

34. Marshall, L.; Mölle, M.; Fehm, H.; Born, J. Scalp recorded direct current brain potentials during human sleep. Eur. J. Neurosci. 1998, 10, 1167-1178.

35. Rowland, V. Dynamic patterns of brain cell assemblies. IV. Mixed systems. Steady states and their shifts. Behavioral studies. Neurosci. Res. Prog. Bull. 1974, 12, 109-121. 
36. Marczynski, T.J.; Karmos, G. Reward contingent positive variation and patterns of neuronal activity in the visual cortex of the cat. In Multidisciplinary Perspectives in Event-Related Brain Potential Research; Otto, D.A., Ed.; Environmental Protection Agency: Washington, DC, USA, 1978; pp. 36-38.

37. Posner, M.I.; Snyder, C.R.; Davidson, B.J. Attention and the detection of signals. J. Exp. Psychol. 1980, 109, 160-174.

38. Moore, W.H.; Haynes, W.O. A study of alpha hemispheric asymmetries for verbal and nonverbal stimuli in males and females. Brain Lang. 1980, 9, 338-349.

39. Rugg, M.; Dickens, A. Dissociation of alpha and theta activity as a function of verbal and visuospatial tasks. Electr. Clin. Neuro 1982, 53, 201-207.

40. Trimmel, M.; Groll-Knapp, E.; Haider, M. Different storage methods for biopotential skin electrodes (sintermetallic $\mathrm{Ag} / \mathrm{AgCl}$ ) and their influence on the bias potential. Eur. J. Appl. Physiol. 1982, 50, 105-116.

41. Seyfried, H. Lern- und Merkfähigkeitstest (LMT); Ketterl: Wien, Austria, 1990.

42. Schoppe, K.J. Verbaler Kreativitätstest-Ein Verfahren zur Erfassung verbal-produktiver Kreativitätsmerkmale; Verlag für Psychologie: Göttingen, Germany, 1975.

43. Torrance, E.P. Torrance Test of Creative Thinking; Scholastic Testing Service, Inc.: Bensenville, IL, USA, 1974.

44. Hennighausen, E.; Heil, M.; Rösler, F. A correction method for DC drift artifacts. Electr. Clin. Neuro 1993, 86, 199-204.

45. Haider, M.; Groll-Knapp, E.; Trimmel, M. Cortical DC-shifts related to sustained sensory stimulation and motor activity. In From Neuron to Action; Deecke, L., Eccles, J.C., Mountcastle, V.B., Eds.; Springer-Verlag: Berlin, Germany, 1990; pp. 59-64.

46. Ghatan, P.H.; Hsieh, J.C.; Petersson, K.M.; Stone-Elander, S.; Ingvar, M. Coexistence of attention-based facilitation and inhibition in the human cortex. Neuroimage 1998, 7, 23-29.

47. Purser, J.; Radford, A.N. Acoustic noise induces attention shifts and reduces foraging performance in three-spined sticklebacks (Gasterosteus aculeatus). PLoS One 2011, 6, doi:10.1371/journal.pone.0017478.

48. Clark, C.; Sörqvist, P. A 3 year update on the influence of noise on performance and behavior. Noise Health 2012, 14, 292-296.

49. Sörqvist, P.; Nöstl, A.; Halin, N. Disruption of writing processes by the semanticity of background speech. Scand. J. Psychol. 2012, 53, 97-102.

50. Valente, D.L.; Plevinsky, H.M.; Franco, J.M.; Heinrichs-Graham, E.C.; Lewis, D.E. Experimental investigation of the effects of the acoustical conditions in a simulated classroom on speech recognition and learning in children. J. Acoust. Soc. Am. 2012, 131, 232-246.

51. Haines, M.M.; Stansfeld, S.A.; Job, R.F.S.; Berglund, B.; Head, J. Chronic aircraft noise exposure, stress responses, mental health and cognitive performance in school children. Psychol. Med. 2001, 31, 265-277.

52. Lercher, P.; Evans, G.W.; Meis, M. Ambient noise and cognitive processes among primary schoolchildren. Environ. Behav. 2003, 35, 725-735.

53. Hygge, S. Classroom experiments on the effects of different noise sources and sound levels on long-term recall and recognition in children. Appl. Cogn. Psychol. 2003, 17, 895-914. 
54. Rösler, F.; Heil, M.; Glowalla, U. Monitoring retrieval from long-term memory by slow event-related brain potentials. Psychophysiology 1993, 30, 170-182.

55. Brunia, C.H.; Damen, E.J. Distribution of slow brain potentials related to motor preparation and stimulus anticipation in a time estimation task. Electr. Clin. Neuro 1988, 69, 234-243.

56. Trimmel, M.; Kundi, M.; Binder, E.; Groll-Knapp, E.; Haider, M. Combined effects of mental load and background noise on CNS activity indicated by brain DC potentials. Environ. Int. 1996, 22, 83-92.

57. Trimmel, M.; Kundi, M.; Binder, G. Effects of background noise and mental load on CNS activity indicated by brain DC potentials. Zbl. Hyg. Umweltmed. 1997, 200, 415-416.

58. Andringa, T.C.; Lanser, J.J. How pleasant sounds promote and annoying sounds impede health: A cognitive approach. Int. J. Environ. Res. Public Health 2013, 10, 1439-1461.

59. Wickens, C.D. Multiple resources and mental workload. Hum. Fact. 2008, 50, 449-455.

60. Hommel, B.; Fischer, R.; Lorenza, S.C.; van den Wildenberg, W.P.; Cellini, C. The effect of fMRI (noise) on cognitive control. J. Exp. Psychol. 2012, 38, 290-301.

61. Addison, J.; Dancer, J.; Montague, J.; Davis, P. Ambient noise levels in university classrooms: detrimental to teaching and learning. Percept. Mot. Skills 1999, 89, 649-650.

62. Michalek, A.M.P.; Watson, S.M.; Ash, I.; Ringleb, S.; Raymer, A. Effects of noise and audiovisual cues on speech processing in adults with and without ADHD. Int. J. Audiol. 2014, 53, 145-152.

63. Beattie, R.L.; Lu, Z.-L.; Manis, F.R. Dyslexic adults can learn from repeated stimulus presentation but have difficulties in excluding external noise. PLoS One 2011, 6, doi:10.1371/journal.pone.0027893.

64. Helfer, K.S.; Freyman, R.L. Stimulus and listening factors affecting age-related changes in competing speech perception. J. Acoust. Soc. Am. 2014, 136, 748-759.

65. Wong, P.C.M.; Jin, J.X.; Gunasekera, G.M.; Abel, R.; Lee, E.R.; Dhar, S. Aging and cortical mechanisms of speech perception in noise. Neuropsychologia 2009, 47, 693-703.

66. Mattys, S.L.; Scharenborg, O. Phoneme categorization and discrimination in younger and older adults: A comparative analysis of perceptual, lexical, and attentional factors. Psychol. Aging 2014, $29,150-162$.

(C) 2014 by the authors; licensee MDPI, Basel, Switzerland. This article is an open access article distributed under the terms and conditions of the Creative Commons Attribution license (http://creativecommons.org/licenses/by/4.0/). 